\title{
Management of febrile children under five years in hospitals and health centres of rural Ghana
}

\author{
Jayne Webster ${ }^{1 *}$, Frank Baiden ${ }^{2}$, Justina Bawah², Jane Bruce ${ }^{1}$, Mathilda Tivura ${ }^{2}$, Rupert Delmini ${ }^{2}$, \\ Seeba Amenga-Etego ${ }^{2}$, Daniel Chandramohan ${ }^{1}$ and Seth Owusu-Agyei ${ }^{2}$
}

\begin{abstract}
Background: The case management of febrile children in hospitals' and health centres' pre-roll out of the new WHO policy on parasitological diagnosis was assessed. The delivery of artemisinin combination therapy (ACT) at these two levels of the health system was compared.

Methods: Structured observations and exit interviews of 1,222 febrile children attending five hospitals and 861 attending ten health centres were conducted in six districts of the Brong Ahafo Region of Ghana. Effectiveness of delivery of case management of malaria was assessed. Proportions of children receiving ACT, anti-malarial monotherapy and antibiotics were described. Predictors of: a febrile child being given an ACT, a febrile child being given an antibiotic and of carers knowing how to correctly administer the ACT were assessed using logistic regression models stratified by hospitals and health centres.
\end{abstract}

Results: The system's effectiveness of delivering an ACT to febrile children diagnosed with malaria (parasitologically or clinically) was 31.4 and $42.4 \%$ in hospitals and health centres, respectively. The most ineffective process was that of ensuring that carers knew how to correctly administer the ACT. Overall 278 children who were not given an ACT were treated with anti-malarial monotherapy other than quinine. The majority of these children, 232/278 were given amodiaquine, 139 of these were children attending hospitals and 93 attending health centres. The cadre of health staff conducting consultation was a common predictor of the outcomes of interest. Presenting symptoms and examinations conducted were predictive of being given an ACT in hospitals and antibiotic in hospitals and health centres but not of being given an ACT in health centres. Treatment-seeking factors were predictive of being given an ACT if it was more than seven days since the fever began and an antibiotic in hospitals but not in health centres.

Conclusion: Interventions to improve adherence to negative parasitological tests are needed, together with guidance on dispensing of antibiotics, but improving the education of carers on how to administer ACT will lead to the greatest immediate increase in the effectiveness of case management. Guidance is needed on implementation of the new test-based treatment for malaria policy in health facilities.

\section{Background}

After several decades of presumptive treatment of febrile children for malaria in moderate to high transmission areas, a new global policy was introduced in 2010 for the parasitological diagnosis of malaria [1]. The confirmation of diagnosis of malaria must be implemented within the broader diagnosis and treatment of febrile illness as part of the Integrated Management of Childhood Illness (IMCI) [2-4]. The IMCI diagnostic algorithm provides a

\footnotetext{
* Correspondence: jayne.webster@lshtm.ac.uk

'Disease Control Department, London School of Hygiene and Tropical Medicine, London, UK

Full list of author information is available at the end of the article
}

step-by-step approach to the clinical assessment of a febrile child based on danger signs, symptoms and clinical examinations. The IMCI clinical assessment algorithm has however been poorly implemented in many countries [5-7].

Once a febrile child has been diagnosed, they must be given appropriate treatment for the condition diagnosed and the carer should be given clear instructions on administration of the treatment. Currently the treatment for malaria defined by the national guidelines in sub-Saharan Africa is an artemisinin combination therapy (ACT). All commonly used artemisinin-based combinations, including artesunate-amodiaquine (ASAQ) and artemether 
lumefantrine $(A L)$, even where the first dose of the treatment is given under observation within the health facility, require further doses to be taken at home. Effective treatment therefore requires that the carer understands, remembers and adheres to instructions on how the treatment should be administered to the child.

Whilst the increased access to rapid diagnostic tests (RDTs) has improved the diagnosis of malaria, the differential diagnoses of other childhood febrile illnesses have not advanced. These depend on robust assessment and interpretation of clinical symptoms and signs and therefore rely on the skills of the clinician. However the clinical assessment of febrile children is often incomplete [8]. This lack of reliable differential diagnoses of febrile illnesses has prompted the concern that the use of RDTs for malaria, whilst decreasing the over-administration of anti-malarials, would result in increased use of antibiotics in children found to be negative for malaria parasites [9]. This has been shown to be the case in several study settings [10-14].

In this study, the case management of febrile children in hospitals and health centres was compared using a health system's effectiveness algorithm. This system's effectiveness analysis measures the cumulative effectiveness of all processes and the effectiveness of individual processes in the delivery of case management of febrile children. Adherence and the predictors of adherence to treatment guidelines where children are presumptively and parasitologically diagnosed for malaria are compared. This study was undertaken pre-roll out of the new policy on parasitological diagnosis of malaria and therefore provides a baseline against which future studies on diagnosis and treatment behaviours may be assessed. However, without an assured supply of RDTs, these findings may also be applicable to the programmatic context for long or short periods of time until product supply management chains and sustained funding can be strengthened.

\section{Methods}

\section{Ethics approval}

The study was approved by the Ethics Review Committees of the Ghana Health Service (GHS), the Kintampo Health Research Centre and the London School of Hygiene and Tropical Medicine. Administrative approval was obtained from the respective district and hospital management teams, health workers and carers of children who gave written consent to be observed and interviewed.

\section{Study setting}

The study was conducted in six out of 19 districts of the Brong Ahafo region of Ghana between May and October 2009. These districts were Kintampo North, Kintampo South, Nkoranza North, Nkoranza South and Tain, purposively selected due to their long-standing relationship with Kintampo Health Research Centre. The study setting, design and procedures have been described elsewhere [8]. Malaria transmission is year round in the six study districts and is the major reason for attendance at outpatient departments (OPD).

District hospitals provide primary clinical care for the population living in the catchment area and act as the first level of referral from health centres. The health centre is the main entry point to the health system for clinical care, though services are also available at the community level through the Community-based Health Planning and Services (CHPS) compounds. Health centres provide basic curative, preventative and reproductive health services.

\section{Study design and sampling procedures}

A cross-sectional health facility survey comprising of a health facility audit, structured observations of case management of febrile children and exit interviews with carers was conducted in five hospitals and ten health centres in 2009. The five study district hospitals were selected, with Kintampo North District Hospital being excluded because another study was running at the time with the same target population. The ten health centres were randomly selected from a comprehensive list of health centres in the six districts using probability proportional to size based on OPD attendance in the previous year.

The sample size estimation was based upon the requirements of the health system's effectiveness analysis, which includes a number of sequential processes in the treatment of a child diagnosed with malaria, and being diagnosed with malaria, being prescribed an $\mathrm{ACT}$, and being given the correct dose of the ACT. The estimation included two stages. In the first stage an estimated 556 febrile children were required to measure a range of outcomes with a frequency of $50 \%$ to achieve a $5 \%$ precision with a design effect of 2. In the second stage this sample size was increased to maintain a $5 \%$ precision on the evaluable sample for each sequential process, including an estimated $60 \%$ of children diagnosed with malaria, $70 \%$ prescribed an $\mathrm{ACT}$ and $90 \%$ given the correct dose.

\section{Data collection}

Consent to conduct the study was obtained from the head of each health facility and a written informed consent was obtained from each staff involved in the management of febrile children. Data on their demographic characteristics, professional experience and training were collected using a structured questionnaire. Carers of young children were approached on entering the health facility by study field workers and a written informed consent was obtained for the carers of children meeting the inclusion criteria (child was under five years of age and had a history of fever in the previous two weeks). A field worker 
observed each step in the management process of the child in the OPD using a structured checklist to record actions and communications by the health workers and the carer. An exit interview was conducted with the carer when ready to leave the facility, which included direct questions on the events during the visit, observation of drugs received and assessment of the knowledge of the carer on how to administer the drugs on leaving the facility. On completion of the process with the carer of the first child the field worker then approached the next eligible child and their carer to enter the facility and repeated the process.

Health facility audits were undertaken at each of the hospitals and health centres to assess the context within which the case management procedures took place.

\section{Study definitions}

Case management of malaria in Ghana is assessed in this study based on the national guidelines at the time which stated: "In young children, fever or history of fever in the absence of other causes of fever should be considered malaria and treatment commenced immediately without waiting for laboratory results. This is done in the context of IMCI. Confirmatory testing in this age group is not required, but may be considered where available" [15].

The health system's effectiveness algorithm had the following four steps: (1) received a clinical or parasitological diagnosis of malaria; (2) given any anti-malarial; (3) given an ACT or quinine; and, (4) carer knew how to give ACT. A diagnosis of malaria without a laboratory confirmatory test was defined as clinical malaria and those cases that were positive for malaria by RDT or microscopy were defined as parasitological malaria. Any anti-malarial included ASAQ, AL, artesunate sulphadoxine-pyrimethamine, artesunate, artesunate suppositories, chloroquine, amodiaquine, and quinine. ACT included ASAQ and AL both of which are recommended first-line treatments for malaria according to the national guidelines [15]. Quinine was also defined as an effective drug at this stage on the assumption that this was given to children who were considered to have severe malaria. Effective knowledge of the carer was defined as knowing that the ACT (ASAQ or AL) should be given twice per day for three days. The data source for the first of these processes was health facility records, and for the others, exit interviews with carers.

The intermediate processes for the system's effectiveness algorithm were deemed to be ineffective if less that $80 \%$ of children completed the process. Cumulative effectiveness of the system is defined as the successful completion of all three intermediary steps, that is, children diagnosed with malaria leaving the health facility with an ACT or quinine with a carer who knows how to correctly administer the treatment.

\section{Data management and analysis}

Data were double-entered and validated using EpiData version 3.1 and Stata 11.0 was used for data processing and analysis. Analyses accounted for the survey design and clustering within health facilities. Findings were stratified by hospital and health centre. The effectiveness of each of the individual intermediate processes and the overall cumulative effectiveness of three processes of the system were analysed.

Pearson's design-based F-test was used to assess the significance of the difference in the effectiveness of each process and the cumulative effectiveness for all health facilities combined and then stratified by hospitals, all health centres and health centres with microscopy.

Potential predictors of the three outcomes: a febrile child being given an ACT; a febrile child being given an antibiotic; and, of the carer knowing how to administer the ACT amongst those given one were assessed using a univariate (unadjusted) logistic regression model. Categories of potential predictors included: child characteristics, carer characteristics, health worker characteristics, and health facility processes. Health facility processes included: presenting symptoms, clinical examinations, parasitological tests, treatments given and treatment seeking for the child by the carer. Adjusted Wald tests were used to assess the association between each potential predictor and the three outcomes. Predictors with Odds Ratios (ORs) significant at the $10 \%$ level (p-values <0.1) were included in multivariable logistic regression models [16] for the three outcomes in order to determine the independent effect of each predictor adjusted for the effect of others. The multivariable models used backward elimination and predictors were considered significant at the $5 \%$ level.

\section{Results}

\section{Characteristics of children and carers}

The case management of 1,122 febrile children attending five hospitals and 861 febrile children attending ten health centres was observed and their carers interviewed on exiting the health facilities. Health providers conducting consultations were mainly medical assistants in both hospitals and health centres, but the proportion of children seen by a doctor was higher in hospitals than in health centres (30.1 versus $1.2 \% ; \mathrm{p}=0.0001$ ) (Table 1 ). The characteristics of the febrile children and their presenting symptoms did not vary between hospitals and health centres. However, a higher proportion of children attending hospitals had health insurance (95.5 versus $81.7 \% ; \mathrm{p}=0.004$ ) than those attending health centres. The carers bringing the child to the hospital were more likely to have completed secondary education (53.8 versus $43.2 \% ; \mathrm{p}=0.05)$ and to speak Bono, one of the three major local languages, (84.4 versus 66.7\%), than those bringing febrile children to health centres. 
Table 1 Characteristics of providers, carers and children in hospitals and health centres

\begin{tabular}{|c|c|c|c|c|c|c|c|c|}
\hline \multirow[t]{2}{*}{ Characteristic } & & \multicolumn{3}{|c|}{$\begin{array}{l}\text { Hospitals } \\
(\mathrm{N}=1,122)\end{array}$} & \multicolumn{3}{|c|}{$\begin{array}{l}\text { Health centres } \\
(\mathrm{N}=861)\end{array}$} & \multirow[b]{2}{*}{$\mathbf{p}$} \\
\hline & & $\mathrm{n}$ & $\%$ & $95 \% \mathrm{Cl}$ & $\mathrm{n}$ & $\%$ & $95 \% \mathrm{Cl}$ & \\
\hline \multicolumn{9}{|l|}{ PROVIDERS } \\
\hline \multirow[t]{3}{*}{ Cadre (vital signs and history taking) } & Medical assistant & 22 & 2.0 & $0.06,6.2$ & 3 & 0.3 & $0.07,1.7$ & 0.05 \\
\hline & Nurse & 353 & 31.5 & $11.0,63.0$ & 80 & 9.3 & $4.7,17.7$ & 0.04 \\
\hline & Nurse assistant & 742 & 66.1 & $34.2,88.0$ & 698 & 81.1 & $57.2,93.2$ & 0.4 \\
\hline \multirow[t]{4}{*}{ Cadre (consultation) } & Doctor & 338 & 30.1 & $10.8,60.6$ & 10 & 1.2 & $0.2,7.1$ & 0.0001 \\
\hline & Medical assistant & 537 & 47.9 & $28.7,67.7$ & 416 & 48.3 & $26.2,71.1$ & 1.0 \\
\hline & Nurse & 146 & 13.0 & $5.7,26.9$ & 164 & 19.1 & $11.0,30.9$ & 0.4 \\
\hline & Nurse assistant & 110 & 9.8 & $1.6,42.2$ & 162 & 18.8 & $7.3,40.4$ & 0.5 \\
\hline \multicolumn{9}{|l|}{ CARERS } \\
\hline \multirow[t]{2}{*}{ Sex } & Male & 62 & 5.5 & $2.6,11.4$ & 38 & 4.4 & $3.0,6.4$ & 0.6 \\
\hline & Female & 1,060 & 94.5 & $88.6,97.4$ & 823 & 95.6 & $93.6,97.0$ & 0.6 \\
\hline \multirow[t]{3}{*}{ Age } & $<18$ & 25 & 2.2 & $1.5,3.3$ & 15 & 1.7 & $1.2,2.5$ & 0.3 \\
\hline & $18-30$ & 639 & 57.0 & $50.1,63.5$ & 482 & 56.0 & $51.0,60.8$ & 0.8 \\
\hline & $\geq 31$ & 458 & 40.8 & $34.9,47.1$ & 364 & 42.3 & $37.1,47.7$ & 0.7 \\
\hline \multirow[t]{4}{*}{ Education } & None & 314 & 28.0 & $22.0,34.9$ & 296 & 34.4 & $25.2,44.9$ & 0.3 \\
\hline & Primary & 192 & 17.1 & $14.9,19.5$ & 182 & 21.4 & $18.0,24.6$ & 0.05 \\
\hline & Middle/Secondary & 604 & 53.8 & $49.1,58.5$ & 372 & 43.2 & $34.3,52.6$ & 0.05 \\
\hline & Tertiary & 12 & 1.1 & $0.6,1.9$ & 11 & 1.3 & $0.7,2.3$ & 0.7 \\
\hline \multirow[t]{3}{*}{ Language (speak any of 3 major dialects) } & Twi/Fanti/Asante & 1,021 & 91.0 & $86.1,94.3$ & 757 & 87.9 & $79.6,93.1$ & 0.4 \\
\hline & Bono & 947 & 84.4 & $76.4,90.1$ & 574 & 66.7 & $44.0,83.6$ & 0.06 \\
\hline & Dagarti & 143 & 12.8 & $9.5,16.9$ & 152 & 17.7 & $12.7,24.1$ & 0.1 \\
\hline Relationship to child & Mother & 977 & 87.1 & $81.8,91.0$ & 766 & 89.0 & $86.6,91.0$ & 0.4 \\
\hline \multicolumn{9}{|l|}{ CHILD } \\
\hline \multirow[t]{3}{*}{ Age } & $<12$ months & 155 & 13.81 & $9.6,19.5$ & 166 & 19.3 & $14.2,25.7$ & 0.1 \\
\hline & $12-23$ months & 329 & 29.3 & $26.8,32.0$ & 238 & 27.6 & $24.3,31.3$ & 0.4 \\
\hline & $\geq 24$ months & 638 & 56.9 & $51.2,62.3$ & 457 & 53.1 & $48.2,57.9$ & 0.3 \\
\hline Sex & Male & 602 & 53.9 & $52.2,55.5$ & 454 & 52.8 & $48.0,57.6$ & 0.7 \\
\hline Health insurance & Yes & 1,071 & 95.5 & $90.8,97.8$ & 703 & 81.7 & $68.7,90.0$ & 0.004 \\
\hline \multirow[t]{4}{*}{ Presenting symptoms } & Vomiting & 338 & 30.1 & $27.2,33.2$ & 260 & 30.2 & $26.5,34.2$ & 1.0 \\
\hline & Cough & 406 & 36.2 & $25.9,48.0$ & 243 & 28.2 & $23.4,33.6$ & 0.2 \\
\hline & Diarrhoea & 303 & 27.0 & $24.2,30.0$ & 268 & 31.1 & $26.9,35.8$ & 0.1 \\
\hline & Abdominal pain & 213 & 19.0 & $15.2,23.4$ & 147 & 17.1 & $13.7,21.0$ & 0.5 \\
\hline
\end{tabular}

Note - languages were those that were coded as speak 'very well' or 'good' but not is the response was speak 'not so well' or 'not at all'.

\section{Health system's effectiveness}

The majority of febrile children who had a clinical or parasitological diagnosis of malaria were appropriately dispensed either an ACT or quinine (88.7 and $85.8 \%$ in hospitals and health centres, respectively) (Figure 1). The most ineffective intermediate process was the carers' knowledge of how to correctly administer ACT to their children. Only $37.6 \%$ of those whose child was given an ACT in hospitals and $51.5 \%$ of those given an $\mathrm{ACT}$ in health centres knew for how many days and how many times per day they should administer the treatment to the child. This resulted in cumulative health system's effectiveness of 31.4 and $42.4 \%$ in hospitals and health centres, respectively.

\section{Malaria tests and prescribing of ACT and antibiotics}

Only a small proportion (10.2\%) of febrile children were tested for malaria parasites in all health facilities; $(12.2$ and $7.6 \%$ in hospitals and health centres, respectively). Whilst all five hospitals had a functioning laboratory and 


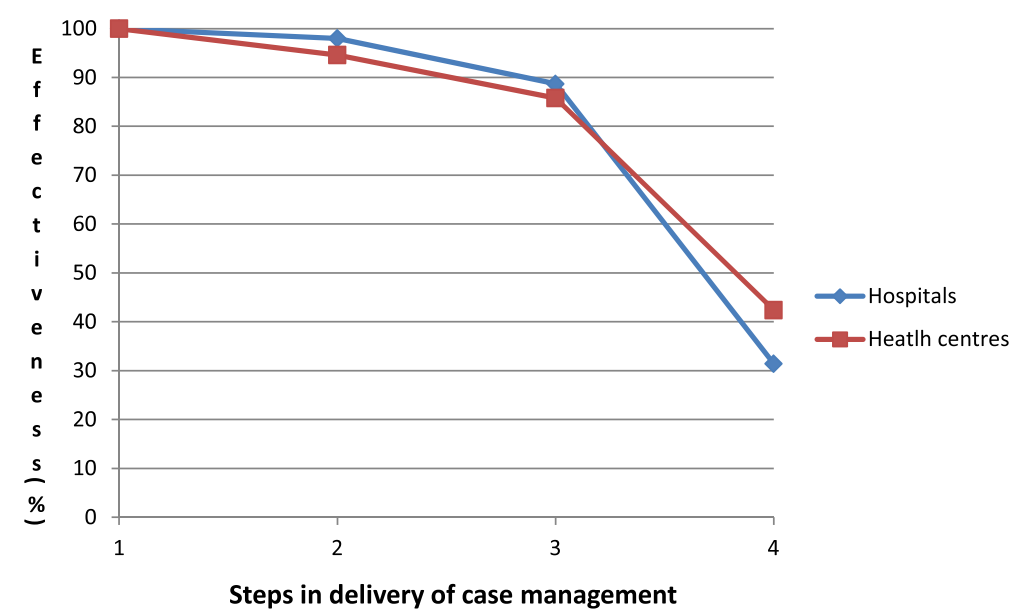

Figure 1 Health systems effectiveness of case management of malaria in febrile children $<5$ years. Note: Step $1=$ Receive a clinical diagnosis or are malaria parasite positive (1065 hospitals, 839 health centres); Step 2 = Given any anti-malarial (1044/1065 hospitals, 794/839 health centres); Step 3 = Given an ACT or quinine (945/1044 hospitals, 720/794 health centres); Step $4=$ Carer knows how to give ACT [number of days and number of times per day]. (334/888 hospitals, 356/692 health centres).

microscopes, this infrastructure was available in only two of the ten health centres. In total, just four RDTs for malaria were conducted in one of the hospitals, although five out of five of the hospitals and nine out of the ten health centres reported having used RDTs previously. Most of the children with a parasitological diagnosis of malaria were given an anti-malarial drug (95\% in the hospitals and $98 \%$ in the health centres). However, the proportion of children with a parasitological diagnosis of malaria receiving ACT was lower in the hospitals than in health centres (67 versus 93\%; $\mathrm{p}=0.01$ ) (Table 2). The likelihood of being given an antibiotic did not differ between hospitals and health centres across any of the different diagnosis groups, although numbers of children in parasite test positive and parasite test negative groups were small.

Although more parasite-positive children were given quinine in health centres than in hospitals (10.8 versus $18.2 \% ; \mathrm{p}=0.08)$ the difference was not statistically significant. Overall, 278 children who were not given an $\mathrm{ACT}$ were treated with anti-malarial monotherapy other than quinine. The majority of these children, 232/278, were given amodiaquine; 139 of these were children attending hospitals and 93 attending health centres. A high proportion of the children given such amodiaquine

Table 2 Diagnosis and treatment with artemisinin combination therapy and antibiotics for febrile children in hospitals and health centres

\begin{tabular}{|c|c|c|c|c|c|c|c|c|}
\hline \multirow[t]{2}{*}{ Characteristic } & & \multicolumn{3}{|c|}{$\begin{array}{l}\text { Hospitals } \\
(n=1122)\end{array}$} & \multicolumn{3}{|c|}{$\begin{array}{l}\text { Health centres } \\
\qquad(n=861)\end{array}$} & \multirow[b]{2}{*}{$\mathbf{p}$} \\
\hline & & $n$ & $\%$ & $95 \% \mathrm{Cl}$ & $\mathrm{n}$ & $\%$ & $95 \% \mathrm{Cl}$ & \\
\hline & Had a parasitological test & 137 & 12.2 & $5.4,25.3$ & 65 & 7.6 & $1.3,34.0$ & 0.58 \\
\hline \multirow[t]{4}{*}{ Test was positive } & & 82 & 7.3 & $3.3,15.5$ & 44 & 5.1 & $1.0,22.2$ & 0.67 \\
\hline & Given an ACT & 55 & 67.1 & $54.4,77.7$ & 41 & 93.2 & $77.4,98.2$ & 0.013 \\
\hline & Given quinine & 9 & 10.8 & $6.5,17.5$ & 8 & 18.2 & $12.7,25.4$ & 0.08 \\
\hline & Given an antibiotic & 5 & 6.1 & $3.2,11.4$ & 4 & 9.1 & $4.0,19.6$ & 0.38 \\
\hline \multirow[t]{4}{*}{ Test was negative } & & 55 & 4.9 & $2.1,11.1$ & 21 & 2.4 & $0.3,15.4$ & 0.48 \\
\hline & Given an ACT & 28 & 50.9 & $38.1,63.6$ & 13 & 61.9 & $51.9,71.0$ & 0.15 \\
\hline & Given quinine & 14 & 25.0 & $10.8,47.9$ & 11 & 52.4 & $43.5,61.1$ & 0.03 \\
\hline & Given an antibiotic & 11 & 20.0 & $6.7,46.6$ & 8 & 38.1 & $27.2,80.3$ & 0.15 \\
\hline \multirow[t]{4}{*}{ Clinical diagnosis } & & 984 & 87.8 & $74.7,94.6$ & 796 & 92.5 & $66.0,98.7$ & 0.58 \\
\hline & Given an ACT & 833 & 84.7 & $74.1,91.5$ & 651 & 81.8 & $67.4,90.7$ & 0.66 \\
\hline & Given quinine & 199 & 20.2 & $12.3,31.4$ & 96 & 12.1 & $9.5,15.1$ & 0.06 \\
\hline & Given an antibiotic & 275 & 28.0 & $12.5,51.3$ & 302 & 37.9 & $27.1,50.1$ & 0.40 \\
\hline
\end{tabular}


monotherapy were those who were clinically diagnosed (195/232). However, 16 of the 27 children found to be malaria parasite positive in hospitals who were not given an ACT, were given amodiaquine monotherapy.

Collating data from all health facilities, including both hospitals and health centres, febrile children were less likely to be given either an ACT or an antibiotic if they were tested than if they were not tested (68.5 versus 83.4\%, $\mathrm{p}=0.02$ for ACT; 13.8 versus $32.4 \%, \mathrm{p}=0.007$ for antibiotic, respectively) (Table 3 ). Children accessing hospitals were also less likely to receive an ACT if they were tested compared with not tested (60.9 versus 84.7\%; $\mathrm{p}=0.006$ ), but this was not the case for children accessing health centres (84.6 versus $81.8 \% ; \mathrm{p}=0.6$ ). In health centres, children tested for malaria were less likely to receive an antibiotic than those who were not tested (18.5 versus $37.9 \% ; \mathrm{p}=0.003)$ but a similar trend in hospitals was not statistically significant (11.6 versus 28.0\%; $\mathrm{p}=0.1$ ).

Febrile children across all health facilities were more likely to be given an ACT if they were malaria parasite positive than if they were tested and found to be parasite negative (76.2 versus $55.8 \% ; \mathrm{p}=0.03)$ and less likely to be given an antibiotic (7.1 versus $24.7 \%$; $\mathrm{p}=0.01$ ) if parasite positive. When stratifying by hospitals and health centres, the trend was similar but not statistically significant in hospitals and health centres, probably due to low numbers.

\section{Predictors of receiving an ACT}

According to the final model, febrile children taken to a hospital were more likely to receive an ACT if they were $\geq 12$ months of age compared to children $<12$ months, their carer spoke one of the three major local dialects and if they had already got medicines from a licensed chemical seller (LCS) (adjusted OR 1.34 95\% CI 1.14, 1.59; p = 0.002) (Table 4). Febrile children taken to hospitals were less likely to be given an ACT if they presented with a cough (adjusted OR 0.50 95\% CI 0.42, 0.60; p < 0.0001), their abdomen was examined (adjusted OR 0.54 95\% CI $0.33,0.89 ; \mathrm{p}=0.02$ ), it was more than seven days since the fever began (adjusted OR 0.08 95\% CI 0.03, 0.22; p = $0.0006)$ and if and if they had already been given medicines at another health facility (adjusted OR $0.44 .95 \%$ CI 0.33, 0.60; $\mathrm{p}=<0.0001$ ).

In health centres, children were more likely to receive an $\mathrm{ACT}$ if the person taking their history and vital signs was a medical assistant or a nurse assistant (medical assistant: adjusted OR 1.84 95\% CI 12.02; nurse assistant: adjusted OR $6.3695 \%$ CI 2.90, 13.95; $\mathrm{p}=0.006$ ) and if they were given quinine (adjusted OR 2.09 95\% CI 1.15, 3.77). Conversely, children attending health centres were less likely to be given an ACT if their carer was their mother (adjusted OR $0.4495 \%$ CI $0.30,0.65 ; \mathrm{p}=0.0005$ ) and if they tested negative for malaria parasites (adjusted OR 0.49 95\% CI 0.27, 0.89; $\mathrm{p}=0.02$ ).

\section{Predictors of receiving an antibiotic}

In the final model, febrile children attending a hospital were more likely to be given an antibiotic if they were seen in consultation by a nurse or health assistant than medical assistant (nurse: adjusted OR2.02 95\% CI 1.55, 2.64, health assistant: adjusted OR 5.94 95\% CI 3.10, 11.40; $\mathrm{p}=0.0001)$ if they had presented symptoms of diarrhoea

Table 3 Treatment received by children tested and not tested for malaria and the result of the test in hospitals and health centres

\begin{tabular}{|c|c|c|c|c|c|c|c|c|c|c|c|c|}
\hline \multirow[t]{2}{*}{$\begin{array}{l}\text { Treament } \\
\text { given }\end{array}$} & \multicolumn{3}{|c|}{$\begin{array}{l}\text { Tested } \\
\mathrm{N}=\mathbf{2 0 3}\end{array}$} & \multicolumn{3}{|c|}{$\begin{array}{l}\text { Not Tested } \\
N=1,780\end{array}$} & \multicolumn{3}{|c|}{$\begin{array}{l}M P+\text { ve } \\
N=126\end{array}$} & \multicolumn{3}{|c|}{$\begin{array}{l}\text { MP -ve } \\
N=77\end{array}$} \\
\hline & $\bar{n}$ & $\%$ & $95 \% \mathrm{Cl}$ & $\bar{n}$ & $\%$ & $95 \% \mathrm{Cl}$ & $n$ & $\%$ & $95 \% \mathrm{Cl}$ & $\bar{n}$ & $\%$ & $95 \% \mathrm{Cl}$ \\
\hline \multicolumn{13}{|l|}{ All HF } \\
\hline AM & 191 & 94.1 & $85.6,97.7$ & 1,719 & 96.6 & $92.3,98.5$ & & & & & & \\
\hline $\mathrm{ACT}$ & 139 & 68.5 & $53.1,80.7$ & 1,484 & 83.4 & $75.1,89.4^{*}$ & 121 & 96.0 & $82.5,99.2$ & 70 & 90.9 & $66.4,98.1$ \\
\hline$A B$ & 28 & 13.8 & $8.0,22.7$ & 577 & 32.4 & $20.7,46.9^{* *}$ & 96 & 76.2 & $52.2,90.4$ & 43 & 55.8 & $42.0,68.9^{*}$ \\
\hline \multicolumn{13}{|l|}{ Hospitals } \\
\hline AM & 127 & 92.0 & $77.8,97.4$ & 967 & 98.3 & $93.9,99.5^{* *}$ & 78 & 95.1 & $59.0,99.6$ & 49 & 87.5 & $49.1,98.1$ \\
\hline $\mathrm{ACT}$ & 84 & 60.9 & $48.2,72.2$ & 833 & 84.7 & $68.6,93.4^{* *}$ & 55 & 67.1 & $49.0,81.2$ & 29 & 51.8 & $34.3,68.9^{*}$ \\
\hline$A B$ & 16 & 11.6 & $3.6,31.3$ & 275 & 28.0 & $8.7,61.1$ & 5 & 6.1 & $2.6,13.6$ & 11 & 19.6 & $3.9,59.6^{*}$ \\
\hline \multicolumn{13}{|c|}{ Health centres } \\
\hline AM & 64 & 98.5 & $80.5,99.9$ & 752 & 94.5 & $85.4,98.0$ & 43 & 97.7 & $15.4,100$ & 21 & 100 & - \\
\hline $\mathrm{ACT}$ & 55 & 84.6 & $80.1,88.3$ & 651 & 81.8 & $66.2,91.2$ & 41 & 93.2 & $46.4,99.5$ & 14 & 66.7 & $54.0,77.3$ \\
\hline$A B$ & 12 & 18.5 & $16.0,21.3$ & 302 & 37.9 & $26.4,51.0^{* *}$ & 4 & 9.1 & $1.7,37.2$ & 8 & 38.1 & $25.8,52.2^{*}$ \\
\hline
\end{tabular}

Note: $A M=$ anti-malarial; $A C T=$ artemisinin combination therapy; $A B=$ antibiotic; $H F=$ health facilities; $H C=$ health centre. ${ }^{*} \mathrm{P}=<0.05 ;{ }^{* * \mathrm{P}}=<0.01$. 
Table 4 Predictors of receiving any artemisinin combination therapy in hospitals and health centres (final models)

\begin{tabular}{|c|c|c|c|c|c|c|}
\hline \multirow{2}{*}{$\begin{array}{l}\text { POTENTIAL } \\
\text { PREDICTORS }\end{array}$} & \multirow[t]{2}{*}{ n } & \multirow[t]{2}{*}{$\%(95 \% \mathrm{Cl})$} & \multicolumn{2}{|c|}{ Unadjusted } & \multicolumn{2}{|c|}{ Adjusted: final model } \\
\hline & & & OR $(95 \% \mathrm{Cl})$ & $p$ & OR $(95 \% \mathrm{Cl})$ & p \\
\hline
\end{tabular}

\section{HOSPITALS}

\section{CHILD: Age}

$\begin{array}{lllc}<12 \text { months } & 115 & 74.2(54.2,87.5) & 1.0 \\ 12-23 \text { months } & 458 & 81.8(67.6,90.6) & 1.56(1.19,2.05) \\ \geq 24 \text { months } & 922 & 83.7(61.4,94.3) & 1.78(1.15,2.76)\end{array}$

0.01

1.0

$1.60(1.16,2.20)$

$1.75(1.25,2.46)$

CARER: Speak any of 3 major dialects

$\begin{array}{lllc}\text { No } & 77 & 76.2(54.8,89.5) & 1.0 \\ \text { Yes } & 841 & 82.4(63.1,92.8) & 1.45(1.28,1.65)\end{array}$

HEALTH WORKER: Cadre (history taking)

$\begin{array}{lllc}\text { Nurse } & 609 & 82.2(58.0,93.9) & 1.0 \\ \text { Doctor } & 17 & 73.9(33.3,94.1) & 0.61(0.16,2.30) \\ \text { Medical assistant } & 288 & 81.6(68.7,90.0) & 0.96(0.49,1.89) \\ \text { Nurse assistant } & 3 & 75.0(5.9,99.3) & 0.65(0.04,10.60)\end{array}$

CONSULTATIONS/EXAMINATIONS: Asked about or reported cough

$\begin{array}{lllc}\text { No } & 611 & 85.5(68.3,94.1) & 1.0 \\ \text { Yes } & 306 & 75.4(58.0,87.1) & 0.52(0.40,0.67)\end{array}$

0.0001

1.0

$0.58(0.36,0.94)$

0.03

$74.0(50.3,88.9)$

114

TREATMENT SEEKING: Time since fever began

$\begin{array}{llc}\leq 2 \text { days } & 529 & 86.3(64.0,95.7) \\ 3-7 \text { days } & 370 & 80.8(63.4,91.1) \\ >7 \text { days } & 18 & 36.0(9.5,75.0)\end{array}$

TREATMENT SEEKING: Given medicines at other HF

$\begin{array}{lll}\text { No } & 870 & 82.8(65.5,92.4) \\ \text { Yes } & 47 & 67.1(42.4,85.0)\end{array}$

TREATMENT SEEKING: Got medicines at an LCS

$\begin{array}{lll}\text { No } & 593 & 79.2(57.6,91.4) \\ \text { Yes } & 324 & 87.1(72.9,94.4)\end{array}$

2. HEALTH CENTRES

CARER: Mother of child

\begin{tabular}{|c|c|c|}
\hline No & 86 & $90.5(78.9,96.1)$ \\
\hline Yes & 620 & $80.9(66.0,90.3)$ \\
\hline
\end{tabular}

HEALTH WORKER: Cadre (history taking)

$\begin{array}{llc}\text { Nurse } & 556 & 79.7(63.6,89.8) \\ \text { Doctor } & 4 & 100.0 \\ \text { Medical assistant } & 70 & 87.5(76.9,93.7) \\ \text { Nurse assistant } & 76 & 96.2(95.7,96.7)\end{array}$

BLOOD TESTS: Malaria parasite negative

$\begin{array}{lllc}\text { No } & 693 & 82.5(67.8,91.3) & 1.0 \\ \text { Yes } & 13 & 61.9(52.9,70.1) & 0.34(0.20,0.60)\end{array}$

$6.36(2.90,13.95)$

\section{$0.67(0.38,1.18)$}

$0.09(0.03,0.23)$

1.0

$0.43(0.31,0.58)$

1.0

$1.78(1.39,2.27)$

1.0

$0.44(0.30,0.65)$

1.0

$1.79(1.08,2.95)$

$6.47(3.07,13.6)$

0.0005

1.0

$0.68(0.39,1.18)$

$0.08(0.03,0.22)$

1.0

$<0.0001$

0.0002

1.0

$1.34(1.14,1.59)$

0.0004

1.0

0.0005

$0.44(0.30,0.65)$

0.0007

1.0

0.006

$1.84(1.02,3.29)$

0.001

1.0

0.02

$0.49(0.27,0.89)$

0.0006

0.0001

0.002

0.02

$<0.0001$

0.02 
Table 4 Predictors of receiving any artemisinin combination therapy in hospitals and health centres (final models) (Continued)

\begin{tabular}{|c|c|c|c|c|c|c|}
\hline \multicolumn{7}{|c|}{ TREATMENTS: Given quinine } \\
\hline No & 80 & $70.2(48.9,85.3)$ & 1.0 & 0.01 & 1.0 & 0.02 \\
\hline Yes & 626 & $83.8(69.9,92.0)$ & $2.20(1.25,3.86)$ & & $2.09(1.15,3.77)$ & \\
\hline
\end{tabular}

LCS = licensed chemical seller.

(adjusted OR 4.99 95\% CI 3.72, 6.68; $\mathrm{p}=<0.0001$ ) or abdominal pain (adjusted OR 2.31 95\% CI 1.33, 4.00; $\mathrm{p}=0.006)$, or sought treatment or drugs elsewhere first (adjusted OR 1.38 95\% CI 1.06, 1.79; p = 0.02) (Table 5). Children seeking treatment at hospitals were less likely to be given an antibiotic if their history and vital signs were taken by a doctor rather than a nurse (adjusted OR $0.2295 \%$ CI $0.05,1.0 ; \mathrm{p}=<0.0001)$, if they were weighed (adjusted OR 0.14 95\% CI 0.07, 0.29; p = <0.0001) or examined for dehydration (adjusted OR 0.09 95\% CI $0.02,0.34 ; \mathrm{p}=0.002$ ) and if they had a positive malaria test (adjusted OR 0.19 95\% CI 0.08, 0.47; p = 0.002).

There were differences between predictors of febrile children receiving an antibiotic at hospitals and health centres. Predictors of receiving an antibiotic in health centres included having health insurance (adjusted OR 1.90 95\% CI 1.11, 3.23; p = 0.02), presenting with a cough (adjusted OR2.24 95\% CI 1.25, 4.00; $\mathrm{p}=0.01$ ), diarrhoea (adjusted OR 4.17 95\% CI 2.62, 6.64; p = <0.0001), or abdominal pain (adjusted OR 1.82 95\% CI 1.26, 2.62; $\mathrm{p}=0.003$ ), and being given any anti-malarial monotherapy (adjusted OR 2.54 95\% CI 1.31, 4.92; p = 0.03). Being seen by a doctor in consultation (adjusted OR 0.10 95\% CI $0.05,0.22 ; \mathrm{p}<0.0001)$ and having a positive malaria test (adjusted OR 0.26 95\% CI 0.16, 0.41; p = 0.0002) were negative predictors of receiving an antibiotic for febrile children attending health centres.

\section{Predictors of knowing how to give an ACT}

In the final model, carers of febrile children taken to hospitals and given an ACT were more likely to know that the child should be given the treatment twice a day for three days if the child was aged 12-23 months compared to if they were $<12$ months of age (adjusted OR $1.7395 \%$ CI 1.25, 2.39; $\mathrm{p}=0.001$ ), and if they were seen in consultation with a nurse or health assistant compared to a medical assistant (nurse: adjusted OR 2.72 95\% CI 1.05, 7.02, health assistant: adjusted OR 5.88 95\% CI 1.96, 17.6; $\mathrm{p}=0.05$ ) (Table 6). Carers were less likely to know how to correctly administer the ACT to their child if they had health insurance (adjusted OR 0.57 95\% CI 0.35, 0.92; $\mathrm{p}=0.03$ ), the child's history was taken by a medical assistant compared with a nurse and if they had a malaria test.

Amongst carers who took their febrile children to health centres and were given an ACT, the carer was less likely to have the correct knowledge of ACT administration if the child's history and vital signs were taken by a nursing assistant in comparison with a nurse (adjusted OR 0.04 95\% CI 0.01, 0.17; $\mathrm{p}=0.006)$ ), if consultation was conducted by a doctor rather than a medical assistant (adjusted OR $0.1395 \%$ CI 0.03, 0.58; p = 0.006) and if the child had a malaria test which was negative (adjusted OR 0.08 95\% CI 0.01, 0.91; $\mathrm{p}=0.04$ ).

\section{Discussion}

In this study, the treatment of febrile children with anti-malarials and antibiotics was described based upon clinical and parasitological diagnosis comparing with and contrasting those attending hospitals from those attending health centres.

The study had several limitations. Whilst majority of the data was taken from structured exit interviews with carers of febrile children, some of the data including that of presenting symptoms and examinations conducted were based on structured observations. It is possible that both health workers and carers of children may have changed their behaviours because they were being observed. Such Hawthorne effects are well documented $[17,18]$. It was assumed that the behaviours observed here were 'best behaviours' of both the health workers and the carers. As the cumulative system's effectiveness was rather low in the hospitals and health centres, any positive change in behaviour due to Hawthorne effect was assumed to be minimal. The enrolment of children and their carers was based upon the number of observations and interviews that can be conducted per day by the available number of field workers and the selection of children were not random. The time of attendance was not, however, a significant predictor in univariate analyses of receiving an ACT, an antibiotic or of the carer knowing how to administer antibiotics and therefore did not impact on the study findings. A stock-take of ACT and antibiotics was not conducted on the day of the survey in each health facility and this may have impacted on the study findings [19]. However, all hospitals and health facilities had ASAQ or AL in stock on the day of the health facility audit and these audits were undertaken immediately prior to the collection of observation and exit interview data. The cadre of health worker conducting both history and vital signs and doing consultation was recorded; however the individual health worker was not. This was a limitation to the inclusion of further health 
Table 5 Predictors of receiving an antibiotic in hospitals and health centres (final model)

\begin{tabular}{llllll}
\hline POTENTIAL & $\mathrm{n}$ & $\%(95 \% \mathrm{Cl})$ & & & Adjusted \\
& & & OR $(95 \% \mathrm{Cl})$ & $\mathrm{OR}(95 \% \mathrm{Cl})$ & $\mathrm{p}$ \\
\hline
\end{tabular}

1. HOSPITALS

HEALTH WORKER: Cadre (history taking)

$\begin{array}{llcc}\text { Nurse } & 227 & 30.6(7.9,69.4) & 1.0 \\ \text { Doctor } & 1 & 4.3(0.6,27.1) & 0.10(0.02,0.62) \\ \text { Medical assistant } & 62 & 17.6(6.4,40.0) & 0.48(0.12,1.91) \\ \text { Nurse assistant } & 1 & 25.0(2.6,80.8) & 0.76(0.07,8.77)\end{array}$

HEALTH WORKER: Cadre (consultation)

$\begin{array}{llcc}\text { Medical assistant } & 117 & 22.4(10.7,41.0) & 1.0 \\ \text { Doctor } & 45 & 13.4(5.9,27.6) & 0.54(0.20,1.41) \\ \text { Nurse } & 52 & 35.9(16.0,62.1) & 1.94(3.87,13.08) \\ \text { Health assistant } & 74 & 67.3(33.8,89.2) & 7.12(3.87,13.08) \\ \text { Other } & 1 & 100.0 & \end{array}$

\section{PRESENTING SYMPTOMS: Diarrhoea}

$\begin{array}{lccc}\text { No } & 157 & 19.2(4.1,56.9) & 1.0 \\ \text { Yes } & 134 & 44.2(22.0,69.0) & 3.34(1.76,6.39)\end{array}$

PRESENTING SYMPTOMS: Abdominal pain

$\begin{array}{lllc}\text { No } & 219 & 24.1(8.0,53.7) & 1.0 \\ \text { Yes } & 72 & 33.8(9.3,71.7) & 161(0.97,268)\end{array}$

\section{PHYSICAL EXAMINATIONS: Dehydration}

$\begin{array}{cccc}\text { No } & 290 & 26.2(8.6,57.4) & 1.0 \\ \text { Yes } & 1 & 6.7(0.3,64.0) & 0.20(0.05,0.82)\end{array}$

\section{PHYSICAL EXAMINATIONS: weighed}

$\begin{array}{lll}\text { No } & 32 & 76.2(60.4,87.0) \\ \text { Yes } & 259 & 24.0(9.1,50.0)\end{array}$

BLOOD TESTS: Malaria test positive

$\begin{array}{llcc}\text { No } & 286 & 27.5(8.9,59.7) & 1.0 \\ \text { Yes } & 5 & 6.0(2.9,12.2) & 0.17(0.05,0.56)\end{array}$

TREATMENT SEEKING: Sought treatment or drugs elsewhere first

$\begin{array}{lllc}\text { No } & 156 & 24.1(7.5,55.5) & 1.0 \\ \text { Yes } & 135 & 28.5(9.6,59.9) & 1.26(1.01,1.56)\end{array}$

\section{HEALTH CENTRES}

\section{CHILD: Have health insurance}

$\begin{array}{lll}\text { No } & 44 & 27.9(20.6,36.4) \\ \text { Yes } & 270 & 38.4(25.7,53.0)\end{array}$

HEALTH WORKER: Cadre (consultation)

$\begin{array}{ll}\text { Medical assistant } & 142 \\ \text { Doctor } & 1 \\ \text { Nurse } & 65 \\ \text { Health assistant } & 60 \\ \text { Other } & 46\end{array}$

PRESENTING SYMPTOMS: cough

$\begin{array}{llcc}\text { No } & 200 & 32.4(20.8,46.6) & 1.0 \\ \text { Yes } & 114 & 46.9(32.4,62.0) & 1.85(1.07,3.20)\end{array}$

$34.9(20.6,52.6)$

$10.0(10.0,10.0)$

$39.6(20.1,63.2)$

$37.0(25.1,50.9)$

$39.0(25.9,53.9)$

1.0

$0.21(0.11,0.41)$

$1.23(0.59,2.56)$

$1.10(0.58,2.06)$

$1.19(0.51,2.78)$

0.04

0.07

0.0001

1.0

$<0.0001$

$0.10(0.05,0.22)$

$1.05(0.53,2.09)$

$1.05(0.60,1.85)$

$1.40(0.63,3.12)$

1.0

$2.24(1.25,4.00)$

$1.38(1.06,1.79)$

0.02

$1.90(1.11,3.23)$

0.03

0.01
0.001

0.07

0.0001

$<0.0001$

0.006 $2.31(1.33,4.00)$

0.002

$0.09(0.02,0.34)$

1.0

$<0.0001$

$0.14(0.07,0.29)$

$0.19(0.08,0.47)$

0.002

0.02 
Table 5 Predictors of receiving an antibiotic in hospitals and health centres (final model) (Continued)

\begin{tabular}{|c|c|c|c|c|c|c|}
\hline \multicolumn{7}{|c|}{ PRESENTING SYMPTOMS: diarrhoea } \\
\hline No & 163 & $27.5(17.6,40.3)$ & 1.0 & \multirow[t]{2}{*}{$<0.0001$} & 1.0 & \multirow[t]{2}{*}{$<0.0001$} \\
\hline Yes & 151 & $56.3(38.0,73.1)$ & $3.40(2.28,5.09)$ & & $4.17(2.62,6.64)$ & \\
\hline \multicolumn{7}{|c|}{ PRESENTING SYMPTOMS: abdominal pain } \\
\hline No & 248 & $34.7(24.2,47.0)$ & 1.0 & \multirow[t]{2}{*}{0.03} & 1.0 & \multirow[t]{2}{*}{0.003} \\
\hline Yes & 66 & $44.9(27.6,63.5)$ & $1.53(1.06,2.22)$ & & $1.82(1.26,2.61)$ & \\
\hline \multicolumn{7}{|c|}{ BLOOD TESTS: Malaria test positive } \\
\hline No & 310 & $37.9(26.7,50.7)$ & 1.0 & \multirow[t]{2}{*}{0.0003} & 1.0 & \multirow[t]{2}{*}{0.0002} \\
\hline Yes & 4 & $9.1(4.3,18.3)$ & $0.16(0.07,0.34)$ & & $0.16(0.07,0.34)$ & \\
\hline \multicolumn{7}{|c|}{ TREATMENTS: Given any monotherapy } \\
\hline No & 256 & $33.7(21.7,48.3)$ & 1.0 & \multirow[t]{2}{*}{0.04} & 1.0 & \multirow[t]{2}{*}{0.02} \\
\hline Yes & 58 & $57.4(37.1,75.5)$ & $2.56(1.14,5.73)$ & & $2.57(1.15,5.74)$ & \\
\hline
\end{tabular}

worker characteristics other than cadre, which was a predictor of all three outcomes.

Although having a positive malaria test was associated with receiving an $\mathrm{ACT}$, one-third of children parasite positive in hospitals were not given an ACT and two-thirds of these children with confirmed malaria who were not given an $\mathrm{ACT}$ were given amodiaquine monotherapy. One possibility for this practice may be the relative ease of administering a suspension to children as compared to a tablet. Often, even in studies, artesunate-amodiaquine tablets are crushed in sugared syrup to ease administration to children [20] and in the programmatic setting this would require the availability of such a syrup. More than $10 \%$ of children given an ACT in health centres were also given quinine and in hospitals this proportion was approaching 20\%. Further studies particularly qualitative studies are needed to explain and understand this practice of giving amodiaquine monotherapy in this setting and of giving quinine alongside $\mathrm{ACT}$.

A rationale for the introduction of malaria test-based management of febrile children is that this will reduce over treatment with ACT. In this study setting based on the use of microscopy, this was found to be true. It is also the case that a greater proportion of those found to be positive for malaria parasites were given an ACT than those who were found to be negative. However, there is still considerable effort needed to convince clinicians to adhere to recommendations for negative test results. These behaviours, however, were in response to parasitological testing with microscopy rather than RDTs and children with a negative RDT have been shown to be more likely to be prescribed an anti-malarial treatment than children with a negative microscopy for malaria in some settings [21].

Whilst a greater proportion of malaria parasite-negative than malaria parasite-positive children were given an antibiotic, a smaller proportion of those tested compared to those not tested were administered an antibiotic and therefore overall the use of testing could be said to have reduced the administration of antibiotics in this study. This is, however, within the context of only a small proportion of children being tested and the situation may differ with increases in the proportion of children tested as the proportion of negative tests compared to those not tested increases.

There were few overlaps in predictors of children receiving an ACT or antibiotic and the carers knowing the correct dose schedule of the ACT received in hospitals and health centres. Characteristics of the child and the carer were infrequently predictors of the three outcomes of interest in this study. There were three exceptions to these amongst those attending a hospital which were: 1) that children of 12 months and above were more likely to be given an ACT; 2) children whose carer spoke one of the three local dialects were more likely to be given an ACT; and, 3) the carers of children 23 to 23 months were more likely to know how to give the ACT correctly. It is likely that the diversity of attendees and their ethnic backgrounds is greater at the district hospital than at the health centres, which would go some way to explaining why speaking a local dialect is not a predictor of a child receiving an ACT in a health centre. It does not however explain why in a hospital, a child should be more likely to receive an $\mathrm{ACT}$ if the carer speaks the local dialect, except perhaps if the health workers were worried about communicating how the drug should be used. Data to enable analysis by socio-economic indicators was not collected in this study and therefore are not able to determine whether this predictor is confounded by socio-economic status.

Children attending health centres were more likely to receive an antibiotic if they had national health insurance and the carers of children with health insurance were less likely to know how to correctly administer ACT. The proportion of children with health insurance in this study 
Table 6 Predictors of knowing how to give an artemisinin combination therapy in hospitals and health centres (final model)

\begin{tabular}{|c|c|c|c|c|c|c|}
\hline \multirow{2}{*}{$\begin{array}{l}\text { POTENTIAL } \\
\text { PREDICTORS }\end{array}$} & \multirow[t]{2}{*}{$\mathrm{n}$} & \multirow[t]{2}{*}{$\%(95 \% \mathrm{Cl})$} & \multicolumn{2}{|c|}{ Unadjusted } & \multicolumn{2}{|c|}{ Adjusted } \\
\hline & & & OR $(95 \% \mathrm{Cl})$ & $p$ & OR $(95 \% \mathrm{Cl})$ & p \\
\hline
\end{tabular}

\section{HOSPITALS}

\section{CHILD: Age}

$<12$ months

$12-23$ months

30

$\geq 24$ months

91

$26.1(3.8,76.1)$

$33.8(7.2,77.1)$

$40.3(12.1,76.9)$

215

CHILD: Has health insurance

No 24

Yes

24
312

$54.6(11.0,92.1)$

$35.7(9.2,75.3)$

HEALTH WORKER: Cadre (history taking)

Nurse

310

$50.9(19.8,81.3)$

Doctor

Medical assistant

0

Nurse assistant

25

1

$8.7(1.5,37.4)$

$33.3(0.6,97.6)$

HEALTH WORKER: Cadre (consultation)

Medical assistant

127

Doctor

72

Nurse

56

Health assistant

80

Other

1

1

BLOOD TESTS: Has a malaria test

No

$$
330
$$

6

$39.6(11.1,77.6)$

$7.1(0.8,43.6)$

\section{HEALTH CENTRES}

HEALTH WORKER: Cadre (history taking)

$\begin{array}{lll}\text { Nurse } & 301 & 54.1(25.7,80.1) \\ \text { Doctor } & 3 & 75.0(13.3,98.3) \\ \text { Medical assistant } & 43 & 61.4(18.9,91.6) \\ \text { Nurse assistant } & 10 & 13.2(10.7,16.1)\end{array}$

\section{HEALTH WORKER: Cadre (consultation)}

$\begin{array}{ll}\text { Medical assistant } & 143 \\ \text { Doctor } & 1 \\ \text { Nurse } & 83 \\ \text { Health assistant } & 102 \\ \text { Other } & 28 \\ \text { PRESENTING SYMPTOMS: Lethargy } \\ \text { No } & 9 \\ \text { Yes } & 348 \\ \text { PRESENTING SYMPTOMS: Oedema } \\ \text { No } & 331 \\ \text { Yes } & 26\end{array}$

$42.7(13.4,78.2)$

$12.5(12.5,12.5)$

$65.9(36.8,86.5)$

$72.9(49.8,87.9)$

$28.9(5.8,72.9)$

$69.2(42.7,87.2)$

$50.2(24.2,76.1)$

$49.4(23.4,75.7)$

$72.2(53.4,85.5)$

BLOOD TEST: Malaria test negative

$\begin{array}{llc}\text { No } & 356 & 51.4(25.1,76.9) \\ \text { Test } & 1 & 7.7(0.3,70.8)\end{array}$

1.0

$1.45(0.96,2.18)$

$1.92(1.08,3.39)$

1.0
$0.46(0.19,1.14)$

0.09

0.002

1.0

0.002

$0.09(0.02,0.37)$

$0.48(0.05,4.44)$

1.0

$0.74(0.15,3.64)$

$1.85(0.61,5.65)$

$7.59(2.36,24.40)$

1.0

$0.12(0.03,0.45)$

1.0

$2.54(0.11,60.14)$

$1.35(0.48,3.77)$

$0.13(0.04,0.40)$

1.0

$0.19(0.04,0.83)$

$2.59(0.91,7.37)$

$3.60(0.78,16.72)$

$0.54(0.06,5.29)$

1.0

$0.45(0.25,0.80)$

1.0

$2.66(1.16,6.09)$

1.0

$0.08(0.01,1.65)$
0.004

1.0

0.009

$0.18(0.05,0.60)$

$0.10(0.04,0.29)$

$0.60(0.05,7.31)$

$1.06(0.33,3.50)$

$2.72(1.05,7.02)$

$5.88(1.96,17.6)$

0.004

1.0

0.006

$7.27(0.05,>100.0)$

$1.45(0.56,3.74)$

$0.04(0.01,0.17)$

0.0001

1.0

0.006

$0.13(0.03,0.58)$

$2.30(0.82,6.44)$

$3.65(0.80$, 16.7)

$5.12(0.60,43.7)$

0.01

1.0

0.006

$0.40(0.22,0.73$

0.02

1.0

0.04

$2.67(1.03,6.92)$

0.09

1.0

0.04

$0.08(0.01,0.91)$ 
population was very high (89\%) overall and was higher for hospitals than health centres, 96 versus $82 \%$. Further studies are needed to understand the influence of the increasing uptake of national health insurance on the prescribing and possible over-administration of antibiotics. It is possible that carers whose children are administered both ACT and antibiotics are less able to remember how to take ACT due to confusion between the two drugs.

The cadre of health worker conducting the consultation was a common predictor of the outcomes of interest in this study; as doctors in this setting are less likely to over-prescribe both ACT and antibiotics than are lower level cadres of health workers but less likely to ensure the understanding of the carer on how to administer the ACT.

Treatment-seeking factors were more influential in the management of febrile children in hospitals than in health centres particularly in terms of previous treatment seeking for the current febrile episode. Children were less likely to be given an ACT if it was more than seven days since their fever began and if they had already been given medicines at another health facility, but more likely to be given an ACT if they had previously taken a medicine from an LCS. This suggests a perception of hospital prescribers that children were likely to have already been given an ACT if they had previously attended another health facility but not if they had got medicine from and LCS.

As assessed using the health system's effectiveness approach, the most ineffective process in administering an ACT in this study was that of ensuring that the carer of the child knew how to administer the ACT correctly. This approach provides a quantitative comparison of the proportion of children for whom each defined process in delivering effective case management is achieved. The findings were not disaggregated by categories of diagnosis in this system's analysis where the focus was on the overall picture. Subsequent disaggregation of the findings by diagnosis showed that the aggregated data masked some issues in treatment with amodiaquine and dispensing of both ACT and quinine for a single febrile episode. These two analytical approaches were used effectively to 1 ) determine the overall quantitative process problems and then, 2) to illuminate more deeply upon treatment based upon diagnosis.

The efficacy of the ACT depends upon adherence to its correct administration and this in turns depends upon the carer's knowledge of the correct administration. Adherence can be improved by interventions focussing on provider knowledge and behaviour [22]. However, more research using qualitative methodologies is required in this study setting to understand the barriers to carers' knowledge and how these can be mitigated within the hospital and health centre settings.
Less than $15 \%$ of children were tested for malaria parasites in the five district hospitals included in the study, the rest were clinically diagnosed. The behaviour and process changes required for hospitals and their staff to provide a parasitological test to all febrile children is quite different to that in health centres in which microscopy has not been available in the past. In hospitals, health workers make decisions on the need for a malaria test, generally by microscopy. A guideline of all febrile patients to be tested for malaria would require that they changed this practice. This policy is likely to be less effectively implemented by higher level cadres such as doctors who have been more highly trained in using their clinical judgement based upon signs and symptoms. In a study in Western Kenya, the proportion of patients given a diagnostic test post introduction of the new parasitological diagnosis guidelines did not increase, but the majority of these tests were by RDT rather than microscopy [23]. More studies are needed on the programmatic implementation of the new parasitological diagnosis of malaria guidelines in hospitals to understand 1) whether all patients are given a parasitological test; 2) the impact of having a parasitological test on overall quality of care; and, 3) the cost and time (for health staff and patients) implications given the generally high patient loads in district hospitals.

\section{Conclusion}

Interventions are needed to improve case management of febrile children in hospitals and health centres. Nonadherence to malaria microscopy negative results is a problem in both hospitals and health centres with microscopy and prescribing anti-malarial monotherapy for parasitepositive children is not uncommon in hospitals. A high proportion of carers of children did not know how to administer ACT correctly, and this problem is more acute in hospitals than health centres. This suggests that education on drug administration that carers receive in the health facilities is inadequate for its purpose. Predictors of being given an ACT, given an antibiotic and of the carer knowing how to administer the ACT are different in hospitals and health centres and therefore different interventions are need to improve case management of febrile children at these two levels of the health system. Studies to direct appropriate and effective interventions in hospitals are particularly needed.

\section{Competing interests}

The authors have declared that there are no competing interests.

\section{Authors' contributions}

JW conceived and designed the study, analysed the data and drafted the manuscript. FB conceived and designed the study, implemented the study, and critically reviewed the manuscript. JuB, MT, RD and SA-E implemented the study, managed the data and critically reviewed the manuscript. JaB analysed the data and critically reviewed the manuscript. DC conceived and designed the study, and critically reviewed the manuscript. SO-A conceived and designed the study, implemented the study, and critically reviewed the manuscript. All authors read and approved the final manuscript. 


\section{Acknowledgements}

We would like to thank the study participants and their carers; the KHRC field team and staff; the Ghana Health Service staff in Kintampo North, Kintampo South, Nkoranza North, Nkoranza South and Tain districts; the Ethics Boards of KHRC, Ghana Health Service and London School of Hygiene and Tropical Medicine, UK

The study was funded by a grant from the Bill and Melinda Gates Foundation as part of the ACT Consortium (www.actconsortium.org). The funder had no role in the design or conduct of the study, analysis or interpretation of the data, or in the decision to publish.

\section{Author details}

'Disease Control Department, London School of Hygiene and Tropical Medicine, London, UK. ${ }^{2}$ Kintampo Health Research Centre, Kintampo, Ghana.

Received: 19 January 2014 Accepted: 19 June 2014

Published: 9 July 2014

\section{References}

1. WHO: Guidelines for the Treatment of Malaria. 2nd edition. Geneva: World Health Organization; 2010.

2. Gove S: Integrated management of childhood illness by outpatient health workers: technical basis and overview. The WHO working group on guidelines for integrated management of the sick child. Bull World Health Organ 1997, 75(Suppl 1):7-24.

3. WHO/UNICEF: Handbook IMCI Integrated Managment of Childhood Illnesses. Geneva: World Health Organization/United Nations Childrens' Fund; 2005.

4. WHO: Guidelines for the Treatment of Malaria. 2nd edition. Geneva: World Health Organization; 2006.

5. Bryce J, Victora CG, Habicht JP, Vaughan JP, Black RE: The multi-country evaluation of the integrated management of childhood illness strategy: lessons for the evaluation of public health interventions. Am J Public Health 2004, 94:406-415.

6. Horwood C, Vermaak K, Rollins N, Haskins L, Nkosi P, Qazi S: An evaluation of the quality of $\mathrm{IMCl}$ assessments among $\mathrm{IMCl}$ trained health workers in South Africa. PLoS One 2009, 4:e5937.

7. Rowe AK, Onikpo F, Lama M, Cokou F, Deming MS: Management of childhood illness at health facilities in Benin: problems and their causes. Am J Public Health 2001, 91:1625-1635.

8. Baiden F, Owusu-Agyei S, Bawah J, Bruce J, Tivura M, Delmini R, Gyaase S, Amenga-Etego S, Chandramohan D, Webster J: An evaluation of the clinical assessments of under-five febrile children presenting to primary health facilities in rural Ghana. PLoS One 2011, 6:e28944

9. Baiden F, Webster J, Owusu-Agyei S, Chandramohan D: Would rational use of antibiotics be compromised in the era of test-based management of malaria? Trop Med Int Health 2011, 16:142-144.

10. Msellem MI, Martensson A, Rotllant G, Bhattarai A, Stromberg J, Kahigwa E, Garcia M, Petzold M, Olumese P, Ali A, Bjorkman A: Influence of rapid malaria diagnostic tests on treatment and health outcome in fever patients, Zanzibar: a crossover validation study. PLoS Med 2009, 6:e1000070.

11. Bastiaens GJ, Schaftenaar E, Ndaro A, Keuter M, Bousema T, Shekalaghe SA: Malaria diagnostic testing and treatment practices in three different Plasmodium falciparum transmission settings in Tanzania: before and after a government policy change. Malar J 2011, 10:76.

12. Batwala V, Magnussen P, Nuwaha F: Antibiotic use among patients with febrile illness in a low malaria endemicity setting in Uganda. Malar J 2011, 10:377.

13. Nadjm B, Amos B, Mtove G, Ostermann J, Chonya S, Wangai H, Kimera J, Msuya W, Mtei F, Dekker D, Malahiyo R, Olomi R, Crump JA, Whitty C, Reyburn $\mathrm{H}$ : WHO guidelines for antimicrobial treatment in children admitted to hospital in an area of intense Plasmodium falciparum transmission: prospective study. BMJ 2010, 340:c1350.

14. D'Acremont V, Kahama-Maro J, Swai N, Mtasiwa D, Genton B, Lengeler C: Reduction of anti-malarial consumption after rapid diagnostic tests implementation in Dar es Salaam: a before-after and cluster randomized controlled study. Malar J 2011, 10:107.

15. Ministry of Health: Guidelines for Case Management of Malaria in Ghana. Accra: Ministry of Health; 2009.

16. Katz MH: Multivariable Analysis: A Practical Guide for Clinicians and Public Health Researchers. Cambridge: Cambridge University Press; 2011.
17. Rowe SY, Olewe MA, Kleinbaum DG, McGowan JE Jr, McFarland DA, Rochat R, Deming MS: The influence of observation and setting on community health workers' practices. Int I Qual Health Care 2006, 18:299-305.

18. Leonard K, Masatu MC: Outpatient process quality evaluation and the Hawthorne effect. Soc Sci Med 2006, 63:2330-2340.

19. Hensen B, Paintain LS, Shretta R, Bruce J, Jones C, Webster J: Taking stock: provider prescribing practices in the presence and absence of ACT stock. Malar J 2011, 10:218.

20. Oyakhirome S, Potschke M, Schwarz NG, Dornemann J, Laengin M, Salazar CO, Lell B, Kun JF, Kremsner PG, Grobusch MP: Artesunate-amodiaquine combination therapy for falciparum malaria in young Gabonese children. Malar J 2007, 6:29.

21. Reyburn H, Mbakilwa H, Mwangi R, Mwerinde O, Olomi R, Drakeley C, Whitty CJ: Rapid diagnostic tests compared with malaria microscopy for guiding outpatient treatment of febrile illness in Tanzania: randomised trial. BMJ 2007, 334:403.

22. Yeung $\mathrm{S}$, White NJ: How do patients use antimalarial drugs? A review of the evidence. Trop Med Int Health 2005, 10:121-138.

23. Skarbinski J, Ouma PO, Causer LM, Kariuki SK, Barnwell JW, Alaii JA, de Oliveira AM, Zurovac D, Larson BA, Snow RW, Rowe AK, Laserson KF, Akhwale WS, Slusker L, Hamel MJ: Effect of malaria rapid diagnostic tests on the management of uncomplicated malaria with artemether-lumefantrine in Kenya: a cluster randomized trial. Am J Trop Med Hyg 2009, 80:919-926.

doi:10.1186/1475-2875-13-261

Cite this article as: Webster et al:: Management of febrile children under five years in hospitals and health centres of rural Ghana. Malaria Journal 2014 13:261.

\section{Submit your next manuscript to BioMed Central and take full advantage of:}

- Convenient online submission

- Thorough peer review

- No space constraints or color figure charges

- Immediate publication on acceptance

- Inclusion in PubMed, CAS, Scopus and Google Scholar

- Research which is freely available for redistribution

Submit your manuscript at www.biomedcentral.com/submit
C Biomed Central 